\title{
Guinea pig as a model to study the carotid body-mediated chronic intermittent hypoxia effects
}

The ability to sense and respond to changes in oxygen is essentia Nino and Dr Asuncion Rocher along with their research team better understand the physiology and pathophysiology of oxygen sensitivity. In particular, the team employ the use of the guinea pig as a model to explore the mechanisms that mediate longoxygen levels. With its unique oxygen-sensing systems, the invaluable tool to study and understand the pathological effects occurring in sleep apnoea disorder. for survival. Dr Angela Gómezterm effects of exposure to low

ypoxia is the reduction of oxygen supply to tissues to
below normal, physiological levels. Hypoxia can be produced, for example, by exposure to high different oxyen-sensing meveloped to maintain the oxygen supply within cells in response to hypoxia. When the body senses a decrease in oxygen, its strategy is to increase breathing dep and rate (hyperventilation), in addition, the heart beats faster. These strategies are controlled through feedback mechanisms triggered by sensory receptors called chemoreceptors. One of the main chemoreceptors responsible for oxygen-sensing is the carotid body (CB), which monitors and responds to changes in the partial pressure of oxyg

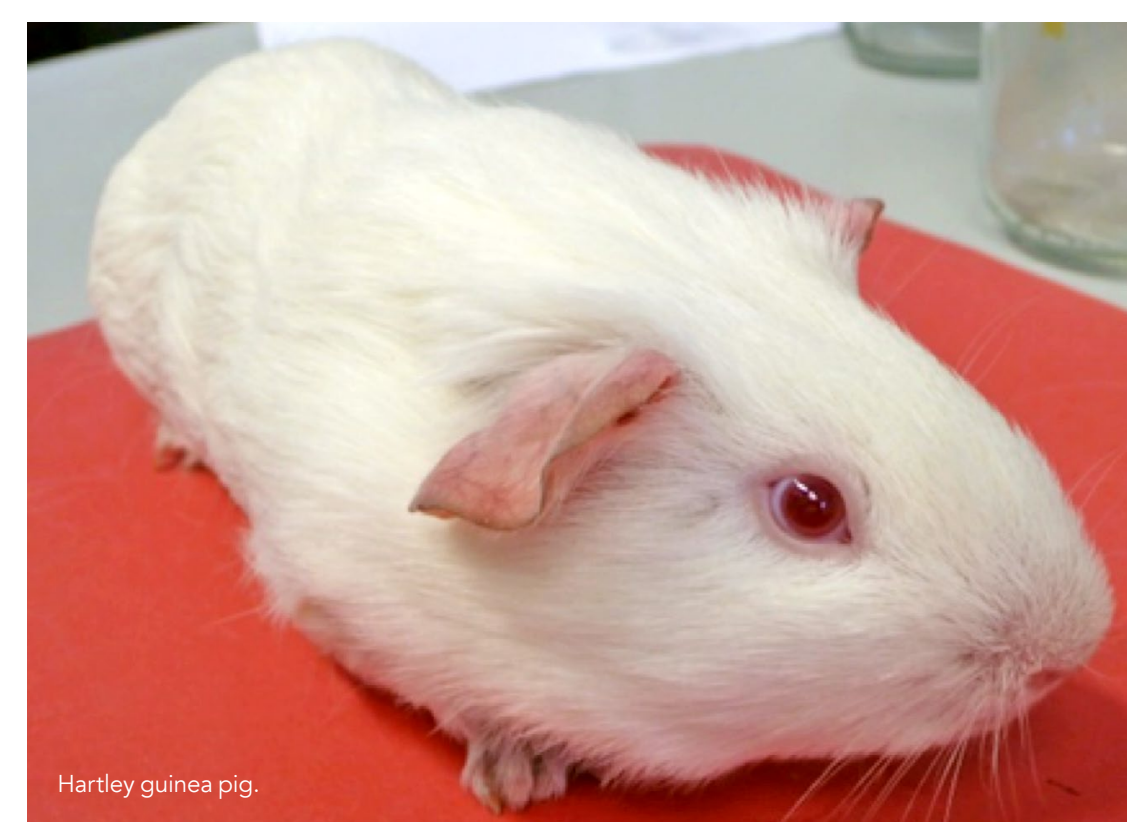

CAROTID BODY CHEMORECEPTORS ARE STIMULATED BY HYPOXIA The $C B$ is found in carotid arteries sides of the throat - and is sensitive to the levels of blood gases. The CB has a high density of blood vessels and is made up of a cluster of specialised cells, called type I cells, that are connected by nerve endings from the carotid sinus nerve a branch of the ninth cranial pair nerve. Upon detecting a drop in blood oxygen levels (or excessive carbon dioxide in the blood, known as hypercapnia), type I cells release specialised signals (known as neurotransmitters, for example, catecholamines, ATP and acetylcholine) that stimulate the carotid sinus nerve, carrying chemoreceptor information to the brainstem to iniliate reflex responses. In this way, the $\mathrm{CB}$ reflex response, triggered by hypoxia, res normal level. Information from the is thus transmitted to the brainstem, which increases breathing frequency (hyperventilation) as well as stimulating the nervous system (sympathetic activation), thereby counteracting the effects of hypoxia.

A research team at the University of Valladolid, led by Dr Angela GómezNino, Professor at the Department of Cell Biology, and Dr Asuncion Rocher Professor of Physiology, explores the mechanisms involved in oxygen
sensing and transduction in $\mathrm{CB}$ arterial sensing and transduction in $\mathrm{CB}$ arteri chemoreceptors. Their research has implications for hypoxia-related pathologies, particularly sleep apnoea
disorder. One exciting focus of their work

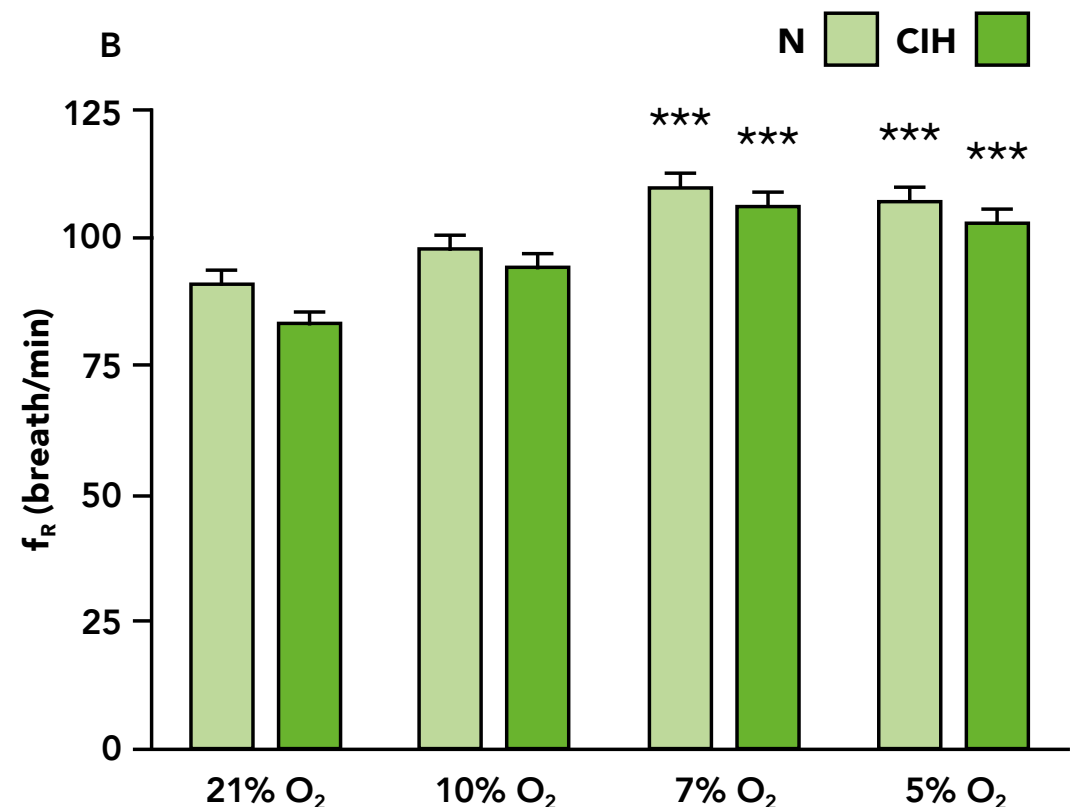

employs the use of the guinea pig. The to help better understand the underlying mechanisms mediating the long-term effects of hypoxia exposure. Specifically, their newly proposed model has mportant implications for und the role of the $C B$ in mediating the pathological effects observed in sleep apnoea disease.

\section{OBSTRUCTIVE SLEEP APNOEA} Sleep apnoea is a serious sleep disorder that occurs when a person's breathing is interrupted during sleep; loud snoring and episodes of breathing interruption during sleep are classic symptoms. In sleep apnoea disorders, may even stop briefly during the seep. In severe cases, the condition causes breathing to repeatedly stop and start during sleep meaning that the brain, and the rest of the body, does not get enough oxygen. Chronic intermittent hypoxia $(\mathrm{CIH})$ is thought to be one of the main causes of arterial high blood pressure observed in obstructive sleep apnoea syndrome. It is believed that repeated episodes of hypoxia/ re-oxygenation produce oxidative stress, inflammation and sympathetic hyperactivity, generating dysfunction of the blood vessel lining (endotheliur and high blood pressure. Indeed, recent evidence suggests a positive responsivess and high blosed

(hypertension), and an increased risk of heart disease.

\section{CHRONIC INTERMITTENT HYPOXIA} Animal models exposed to recurrent hypoxia and re-oxygenation episodes in $\mathrm{CH}$ show increased $\mathrm{CB}$ sensitisation response and chemoreceptor input to the brainstem, exaggerating the resulting nervous reflex (sympathetic reflex). Experiments have shown that if the $C B$ is removed, these effects can be reduced in response to intermitten hypoxia. The majority of hypertension

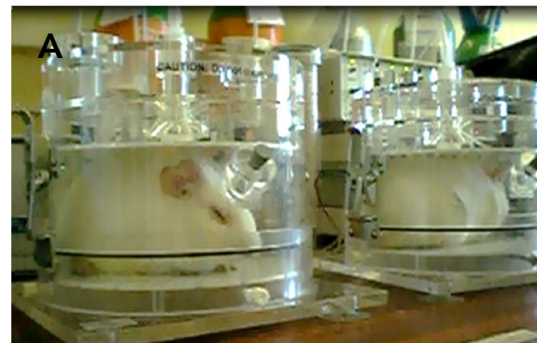

Plethysmography recording of respiratory breathing air and intermittent hypoxia (CIH) exposed guinea pigs. A. Image of awake and
freely moving guinea pigs during whole body

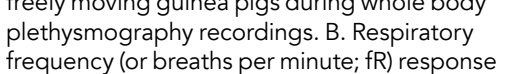
air (21\% oxygen), acure hypoxia (5 min) $\left(5 \% \mathrm{CO}_{2}\right)$ tests. Only intense hypoxia $\left(7 \% \mathrm{O}_{2}\right)$ and

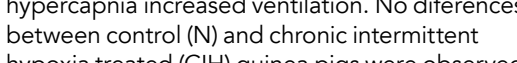
hypoxia treated (NII) guinea pigs were observed.
Data are expressed as mean \pm SEM $(n-8)$ Two-

tongen rodent and

between rodent and cardiovascular responses. In contrast, experiments using guinea pigs, originally from the Andes, showed a different response in early studies led by $\mathrm{Dr}$ Gómez-Niño. Her work demonstrated that guinea pigs show a poor or no ventilatory response to hypox compared to other mammals.

Dr Gómez-Niño's intriguing research demonstrates that unlike other rodents, the poor ventilatory response to hypoxia in guinea pigs is due to low levels

The team are exploring the guinea pig as a model to better understand the mechanisms that mediate long-term effects of exposure to low oxygen levels.

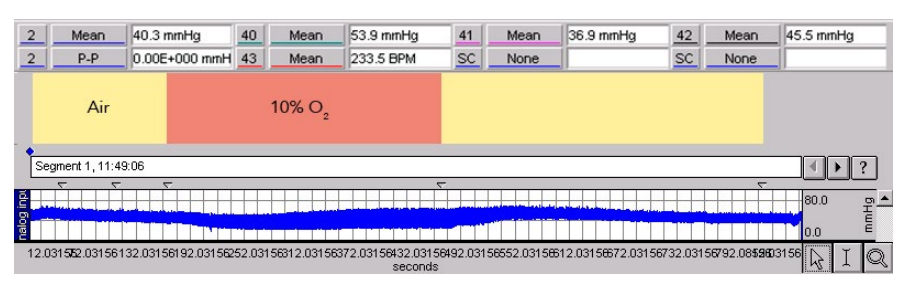

Cardiovascular responses to hypoxia. Continuous recording of arterial blood pressure from a $\mathrm{ClH}$
guinea pig breathing air $(21 \% \mathrm{O})$ ) or hypoxia $(10 \%$ ). To measure arterial pressure, guinea pigs were

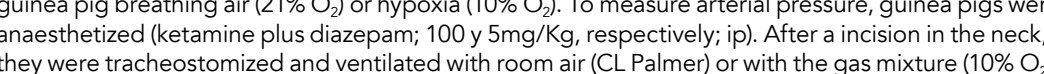
and $90 \%$ N2). Once the right common carotid artery was located, it was cannulated with a catheter connected to a pressure transducer (Transpac IV; ICU Medical, San Clemente, CAA and signals
and stored (BIOPAC Systems, Inc. MP 150, Goleta, CA; Acknowledge 3.9.1) for later analysis. 


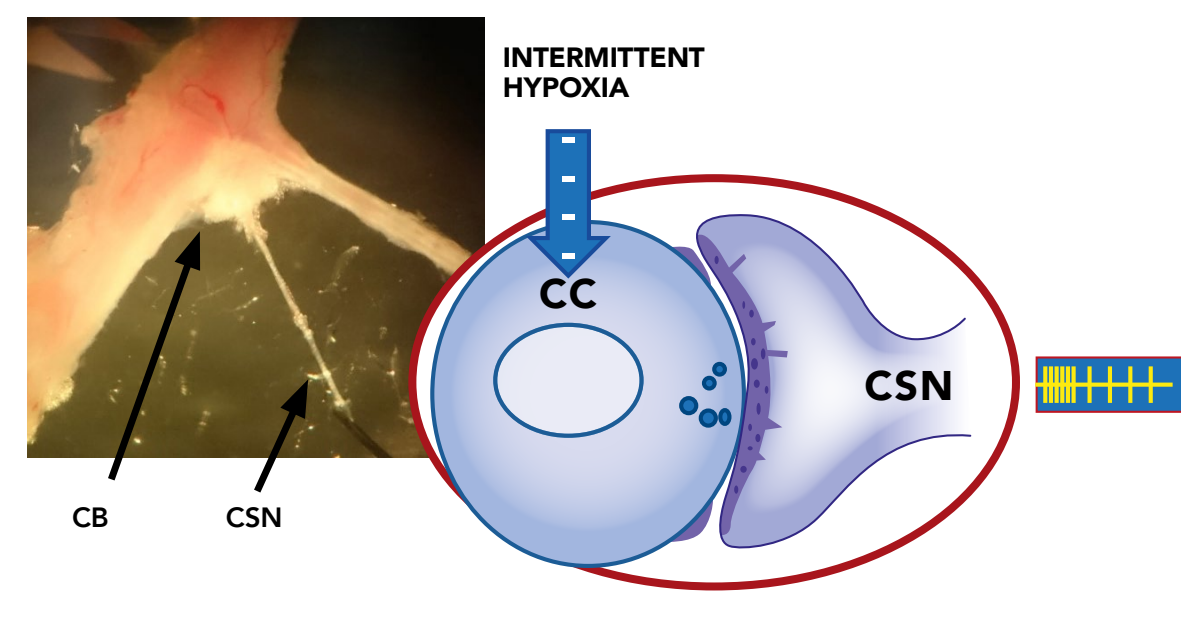

The picture shows the dissection of the bifurcation of the common carotid artery and (hypoxia) is detected by chemoreceptor or type I cells (CC) of the carotid body that by releasing neurotransmitters increase the frequency of action potentials or dischargas in
the carotid sinus nerve (CSN). This information reaches the brainstem generating systemic

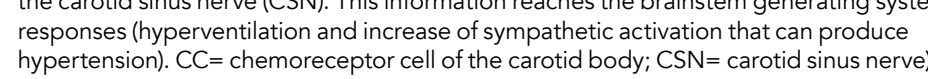

政

The team showed an absence of the hypoxia-driven CB reflex in the guinea pig.

the guinea pig CB response to hypoxia and compared it to the well-known rat hypoxic response. Their research, published in Frontiers in Physiolog, demonstrated for the first time the absence of the hypoxia-driven $\mathrm{CB}$ reflex in the guinea pig. This lack of guinea pig $C B$ response to hypoxia would suppress the chemo-reflex sensitisation, reducing or eliminating the respiratory and nervous reflex (sympathetic reflex) effects of intermittent hypoxia exposure. The lack CIH does not modify the excitability of Che Their research did show, however that intermittent hypoxia-induced

sympathetic hyperactivity and promoted cardiovascular responses by increasing heart rate and arterial blood pressure and that this is independent of $\mathrm{CB}$ stimulation.

\section{INVESTIGATING THE LACK OF} DSPONSE TO HYPOXIA

Dr Gómez-Niño went on to test the ide hat this lack of CB hypoxia response guinea pig would suppress chemoreflex sensitisation, thereby attenuating or eliminating respiratory, sympathetic nd cardiovascular effects of $\mathrm{CIH}$ treatment. The research team set out to explore whether ha guinea pig $\mathrm{CB}$ was to coracte $\mathrm{CH}$ effects on

chemoreceptors with cardiovascular and respiratory responses to hypoxia.

To mimic the situation of an obstructive male guinea pigs to acute hypoxia (30day exposure to $\mathrm{ClH}$ ). They measured CB secretory activity, ventilatory parameters, systemic arterial pressure and sympathetic activity. Their results showed that guinea pigs exposed to $\mathrm{CH}$ lack activty (as measured by release of O) resulted in an increare hypoxia (7\% response and oxygen consumption to the $\mathrm{ClH}$ animals. In other words, $\mathrm{ClH}$ exposure blunted hyperventilation to hypoxia normalised to oxygen consumption. However, the team found that catecholamine levels were increased in the blood, suggesting that CIH induced nerve activity. The team concluded that $\mathrm{ClH}$ does not sensitise the $C B$ chemoreceptor response to hypoxia but promotes cardiovascula ajustments, albert not via the

A MODEL TO INVESTIGATE CHRONIC INTERMITTENT HYPOXIA

Dr Gómez-Niño and Dr Rocher postulate Dibsence of the hypoxia-driven

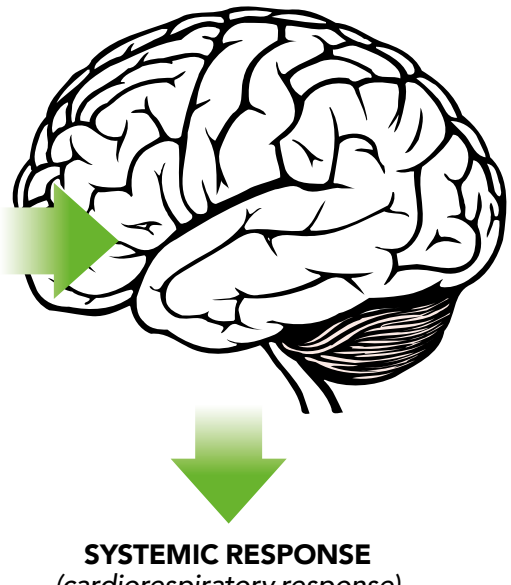

SYSTEMIC RESPONSE
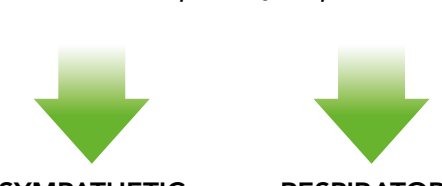

SYMPATHETIC
STIMULATION

RESPIRATORY
RESPONSE

HYPERTENSION HYPERVENTILATION

be similar to that seen in neonatal mammals (In Olea et al., 2018, the team showed that it is not the case). Neonatal mammals have an immature CB chemoreflex and respond to hypoxia through another pathway - via the direct effect of hypoxia on, the adrenal medulla. (th could be through the hypoxic stimulation of specific areas in the central

Excitingly, the researchers at the University of Valladolid believe that the guinea pig represents a usefult tool for examining the mechanisms underlying the long-term effects of $\mathrm{CIH}$ exposure - in particular, the brainstem sensitivity to hypoxia and cardiovascular responses generated by intermittent hypoxia. The team's hope is that this model will also provide evidence for the role of the CB mediating pathological effects in sleep apnoea diseases. Dr Gomez-Nino and Dr Rocher's next steps are to examine the missing mechanisms that underlie the lack of effects of intermittent hypoxia on for gits role in mediating hypertensionce observed in sleep apnoea disorder.

\section{Behind the Research a \\ Dr Ángela Gómez-Niño}

E: angela@biocel.uva.es E: rocher@ibgm.uva.es $\quad T: \pm 34983423086 \quad T: \pm+34983423000$ ext 4122

Research Objectives

DrÁngela Gómez-Niño and Dr Asuncion Rocher along with their research team at Valladolid University aim to better understand the physiology and pathophysiology of oxygen sensitivity. In particular, the team employ the use of the guinea pig as a model to explore the mechanisms that diate long-term effects of exposure to low oxygen levels.

\section{Detail}

DrÁngela Gómez-Niño

Uhe University of Valladolid

47002 Valladolid,

Bio

Dr Ángela Gómez-Niño is associated professor teaching cell biology and her research is focused on the mechanisms involved in oxygen detection and transduction in the caro body arterial chemoreceptors and the hypoxia-related pathologies, mainly those associated to sleep apnoea disorders.

Dr Asuncion Rocher is Professor of Physiology at the Medical School of the Valladolid University where she and biomedicine. Her research is focused on arterial chemoreception, oxygen sensing and hypoxi-related pathologies.

Funding
MINECO/FEDER, UE BFU2015-70616R, ISCiii CIBER CB06/06/0050 and FUNGE UVa PIP-063_166091.

\section{Collaborators}

Elvira Gonzalez-Obeso

- Inmaculada Docio

- Elena Olea

- Ana Obeso

- Jesus Prieto-LLoret

- Teresa Gallego-Martin

\section{References}

Docio I. Olea, E, LLoret J-P, Gallego-Martin T, Obeso A, Gomez-Niño A, Rocher A. (2018). 'Guinea Pig as a Mode to Study the Carotid Body Mediated Chronic Intermittent Hypoxia Effects'. Front. Fhysiol. 9.694. doi. 10.3389/ phys.2018.00694.

Gonzalez-Obeso, E., Docio, I., Olea, E., Cogolludo, A. Obeso, A., Rocher, A., and Gomez-Niño A. (2017). 'Guinea pig oxygen-sensing and carotid body functional properties'. Front. Physiol, 8:285. doi: 10.3389/fphys.2017.00285.

Olea, E., Gonzalez-Obeso, E., Agapito, T., Obeso, A., Rigual, R. Rocher, A., and Gomez-Niño A. (2018). 'Adrenal medulla' chemo sensitivity does not compensate for the lack of hypoxia-driven carotid body chemoreflex in guinea pigs'. Adv. Exp. Med. Biol, 1071: 167-174.

\section{Personal Response}

What first drew you both to the guinea pig as a research?

II Our previous experience working on mammal arterial chemoreceptors was based mainly on rats. We have used the hallmark of seep apnea disorc remitent hypoxia, in patients. The literature showed that guinea pigs did not hyperventilate in hypoxic environments what led us to characterize the guinea pig carotid body. We demonstrated that guinea pigs have a hypo-functional carotid body, hypothesis of the carotid bodyparia. Based in our,

hypothesis of the carotid body as the origin of sleep apnea
symptoms, we decided to use guinea pigs as a model to
study the effects of intermittent hypoxia exposure.

I i 1. diggeres 